\title{
Global regularity for a logarithmically supercritical hyperdissipative dyadic equation
}

\author{
D. Barbato, F. Morandin, and M. Romito \\ Communicated by Terence Tao, received March 12, 2014.
}

\begin{abstract}
We prove global existence of smooth solutions for a slightly supercritical dyadic model. We consider a generalized version of the dyadic model introduced by Katz-Pavlovic $\mathbf{1 0}$ and add a viscosity term with critical exponent and a supercritical correction. This model catches for the dyadic a conjecture that for Navier-Stokes equations was formulated by Tao $\mathbf{1 3}$.
\end{abstract}

\section{Contents}

\begin{tabular}{lll}
1. & Introduction & 39 \\
\hline
\end{tabular}

2. Preliminaries 41

3. The main result 44

$\begin{array}{ll}\text { References } & 52\end{array}$

\section{Introduction}

The a priori estimate of relevant quantities is a crucial part of the analysis of PDEs. For our purposes, the most interesting example is the system of NavierStokes equations in dimension three. In that case the kinetic energy and the energy dissipation are super-critical, hence in a way negligible, quantities with respect to the scaling invariance of the problem. Indeed, proofs of regularity are available only in the so-called hyper-dissipative case, where the Laplace operator is replaced by $(-\Delta)^{\alpha}$ for $\alpha \geq 5 / 4$ and this additional dissipation makes the energy relevant again (see for instance [11, 9]).

In a recent paper Tao [13 has shown that hyper-dissipativity can be slightly relaxed by a logarithmic factor. The idea originates from the same author [12] and

1991 Mathematics Subject Classification. Primary 35; Secondary 76.

Key words and phrases. Dyadic model, global existence, slightly supercritical dyadic model. 
has been applied in other problems, mainly from dispersive equations. In $[\mathbf{1 3}$ Tao adds a small correction to the hyper-dissipative term, replacing $(-\Delta)^{5 / 4}$ with

$$
\frac{(-\Delta)^{5 / 4}}{g\left((-\Delta)^{1 / 2}\right)^{2}}
$$

and provides a simple and neat proof of global existence if $\int 1 /\left(\operatorname{sg}(s)^{4}\right)=\infty$. He then suggests that the same result should hold, based on some heuristics on the flow of energy, under the weaker condition $\int 1 /\left(s g(s)^{2}\right)=\infty$.

The aim of this paper is to prove Tao's conjecture for the dyadic model, a simplified version of the Navier-Stokes equations, that nevertheless has shown to be an effective tool in the understanding of the full Navier-Stokes problem [14. In particular, we believe that the main result of our paper (Theorem 5 gives a complete answer to some questions raised in Remark 5.2 of [14]. As a bonus result, in Section 3.3.1 we prove that the conjecture in $\mathbf{1 3}$ is true for the vector-valued dyadic model introduced in [14. A proof of the conjecture for the full Navier-Stokes equations is a work in progress.

1.1. The model. Given $\beta>0$ and two real sequences $\phi=\left(\phi_{n}\right)_{n \geq 1}$ and $g=\left(g_{n}\right)_{n \geq 1}$, with $\phi$ bounded and $g$ positive, set $k_{n}=2^{\beta n}$ for $n \geq 0$. Consider the critical hyper-dissipative generalized dyadic model,

$$
\left\{\begin{array}{l}
X_{n}^{\prime}=\phi_{n-1} k_{n-1} X_{n-1}^{2}-\phi_{n} k_{n} X_{n} X_{n+1}-\frac{1}{g_{n}} k_{n} X_{n}, \quad t>0, n \geq 1, \\
X_{n}(0)=x_{n},
\end{array}\right.
$$

where $X=\left(X_{n}\right)_{n \geq 0}$ is a family of real functions, $X_{0} \equiv 0$ and $x=\left(x_{n}\right)_{n \geq 1}$ is the given initial condition.

The classical critical regime here corresponds to $g \equiv 1$. Tao's statement for Navier-Stokes equation, transposed on our model, works whenever $\sum_{n} g_{n}^{-2}=\infty$ ( $g_{n}=\sqrt{n}$ for instance), while the conjecture, on our model, states that global regularity should hold for $\sum_{n} g_{n}^{-1}=\infty$ (e. g. $g_{n}=n$ ).

The role of the coefficients $\phi$ is to break the structure of the non-linearity. Otherwise, as shown in 4, if $\phi \equiv 1$, the energy flow is very steady, in the sense that the transfer of energy from $X_{n}$ to $X_{n+1}$ starts before $X_{n-1}$ is discharged enough and this allows to prove regularity in a full supercritical regime. Further generalizations are possible, see Section 3.3 .

The dyadic model has been introduced in [10 and analyzed in several other works $\mathbf{7}, \mathbf{8}, \mathbf{1}, \mathbf{2}$. The model with viscosity has been initially introduced in $\mathbf{6}$ and further analyzed in $[\mathbf{5}, \mathbf{4}$.

1.2. The dyadic version of $\mathbf{1 3}$. It is easy to be convinced that Tao's condition $\int 1 /\left(s g(s)^{4}\right)=\infty$ reads in our case as $\sum 1 / g_{n}^{2}=\infty$. To this end, we reproduce in this section a non-rigorous sketch of the idea of [13] adapted to the dyadic framework. Assume also, as we do, that $\left(g_{n}\right)_{n \geq 1}$ and $\left(k_{n} / g_{n}\right)_{n \geq 1}$ are non-decreasing. Assume moreover, for simplicity, that $g_{n}=g(n)$, where $g$ is non-decreasing, continuous, non-zero on $[0, \infty)$ and $\int g(x)^{-2}=\infty$.

Given a solution $X$, set for $s \geq 1$,

$$
a(t)=\sum_{n=1}^{\infty} \frac{k_{n}}{g_{n}} X_{n}^{2}, \quad A(t)=\sum_{n=1}^{\infty} k_{n}^{2 s} X_{n}^{2}, \quad B(t)=\sum_{n=1}^{\infty} \frac{k_{n}^{2 s+1}}{g_{n}} X_{n}^{2} .
$$


We know by the energy estimate that $a \in L^{1}([0, \infty))$. By differentiating and using the Cauchy-Schwartz and Young inequalities,

$$
\frac{d}{d t} A+2 B=2\left(2^{2 \beta s}-1\right) \sum_{n=1}^{\infty} \phi_{n} k_{n}^{2 s+1} X_{n}^{2} X_{n+1} \leq B+c \sum_{n=1}^{\infty} g_{n} k_{n}^{2 s+1} X_{n}^{2} X_{n+1}^{2} .
$$

Split the sum on the right-hand side in a sum $[\mathrm{L}]$ up to $N$ and in a sum $[\mathrm{H}]$ from $N$ on, where $N$ will be chosen at the end. On the one hand,

$$
[\mathrm{L}]=\sum_{n=1}^{N} g_{n}^{2}\left(\frac{k_{n}}{g_{n}} X_{n}^{2}\right)\left(k_{n}^{2 s} X_{n+1}^{2}\right) \leq c g_{N}^{2} a A,
$$

on the other hand

$$
[\mathrm{H}]=\sum_{n \geq N} \frac{g_{n}}{k_{n}}\left(k_{n}^{s+1} X_{n}^{2}\right)\left(k_{n}^{s+1} X_{n+1}^{2}\right) \leq \frac{g_{N}}{k_{N}} A^{2} .
$$

If we choose $N$ so that $k_{N} \approx A$, that is $N \approx \log A$, we have

$$
\dot{A} \leq c(1+a) g(\log A)^{2} A,
$$

whose solutions stay bounded on bounded sets.

1.3. The dyadic version of Tao's conjecture. We present here a heuristic argument that shows, as in Remark 1.2 of $\mathbf{1 3}$, that the weaker assumption $\sum_{n} g_{n}^{-1}=\infty$ is sufficient for global regularity.

Indeed, let $X$ be a weak solution on $[0, T)$ and consider a blow-up scenario in $T$ : at some time $t$ the energy of solution is concentrated in $n, n+1, \ldots n+m$ and $n \rightarrow \infty$ when $t \rightarrow T$. The balance of energy on $n, \ldots, n+m$ yields:

$$
\frac{d}{d t}\left(\frac{1}{2} \sum_{i=n}^{n+m} X_{i}^{2}\right)=\phi_{n-1} k_{n-1} X_{n-1}^{2} X_{n}-\phi_{m} k_{m} X_{n+m}^{2} X_{n+m+1}-\sum_{i=n}^{n+m} \frac{k_{i}}{g_{i}} X_{i}^{2},
$$

where we could imagine $\phi_{n-1} k_{n-1} X_{n-1}^{2} X_{n}$ as the energy moving from $n-1$ to $n$, $\phi_{m} k_{m} X_{n+m}^{2} X_{n+m+1}$ the energy moving from $n+m$ to $n+m+1$, and $\frac{k_{i}}{g_{i}} X_{i}^{2}$ the energy dissipated in $i$. So, roughly speaking, $k_{n} X_{n}^{3}$ is the speed at which the energy moves from $n$ to $n+1$, whereas $\frac{k_{n}}{g_{n}} X_{n}^{2}$ is the speed at which the energy is dissipated in $n$.

Now in the blow-up scenario, to go to high " $n$ "s, the energy has to go through all the states. The ratio between the energy dissipated and the energy that goes through $n$ is $\frac{1}{g_{n} X_{n}} \geq \frac{C}{g_{n}}$. So, to have a non-trivial amount of energy reaching the infinite state, we have to require $\sum g_{n}^{-1}<\infty$.

Our proof is a rigorous version of the above argument. We find a recursive formula $(9)$ for the tail energy and dissipation. Then we prove that any sequence satisfying the recursion decays super-exponentially fast.

\section{Preliminaries}

\subsection{Basic definitions.}

Definition 1. A weak solution is a sequence of $X=\left(X_{n}\right)_{n \geq 1}$ of differentiable functions on all $[0, \infty)$, satisfying (1). 
Whenever $X$ denotes a weak solution, $E_{n}(t)$ and $F_{n}(t)$ will denote the energy of the tails: for all $n \geq 1$ and $t \geq 0$,

$$
E_{n}(t):=\sum_{i \leq n} X_{i}^{2}(t)<\infty, \quad \text { and } \quad F_{n}(t):=\sum_{i \geq n} X_{i}^{2}(t) \leq \infty .
$$

We will also denote by $E$ the total energy of the solution $X$ : for all $t \geq 0$,

$$
E(t):=\sum_{n \geq 1} X_{n}^{2}(t)=\lim _{n \rightarrow \infty} E_{n}(t)=\|X(t)\|_{H}^{2} .
$$

Clearly $E(t)=E_{n}(t)+F_{n+1}(t)$ for all $n \geq 1$. From (1) we get

$$
\frac{d}{d t}\left(X_{n}^{2}\right)=2 \phi_{n-1} k_{n-1} X_{n-1}^{2} X_{n}-2 \phi_{n} k_{n} X_{n}^{2} X_{n+1}-\frac{2}{g_{n}} k_{n} X_{n}^{2},
$$

so that if $X$ is a weak solution, for all $n \geq 1$,

$$
E_{n}^{\prime}=-2 \phi_{n} k_{n} X_{n}^{2} X_{n+1}-\sum_{i \leq n} \frac{2}{g_{i}} k_{i} X_{i}^{2} .
$$

To compute the variation of $F_{n}$ we need an extra condition on solutions.

Definition 2. A weak solution $X$ satisfies the energy inequality on $[0, T]$ if

$$
E(t)+\int_{0}^{t} \sum_{n \geq 1} \frac{2}{g_{n}} k_{n} X_{n}^{2}(s) d s \leq E(0), \quad t \in[0, T] .
$$

A weak solution satisfies the energy equality if there is equality in the above formula.

We remark that, as is expected in this class of problems, regularity readily implies uniqueness and that the energy inequality holds (there is no anomalous dissipation). The converse is not true in general (see for instance [1, 3, ).

By (2) and (3) it follows that, if $X$ satisfies the energy inequality, then

$$
F_{n}(t) \leq F_{n}(0)+\int_{0}^{t} 2 \phi_{n-1} k_{n-1} X_{n-1}^{2} X_{n} d s-\int_{0}^{t} \sum_{i \geq n} \frac{2}{g_{i}} k_{i} X_{i}^{2} d s .
$$

The following proposition gives a sufficient condition for the energy equality. We first introduce some functional spaces. For all $s \in \mathbb{R}$ and $p \geq 1$, let $W^{s, p}$ denote the Banach space

$$
W^{s, p}=\left\{x=\left(x_{n}\right)_{n \geq 1} \in \mathbb{R}^{\mathbb{N}}:\|x\|_{W^{s, p}}^{p}:=\sum_{n \geq 1} 2^{p s n}\left|x_{n}\right|^{p}<\infty\right\} .
$$

In particular, we set $H^{s}=W^{s, 2}$ and $H:=H^{0}=\ell^{2}(\mathbb{R})$.

Proposition 3. Let $T>0$ and $X$ be a weak solution with initial condition $x \in H$. If $X \in L^{3}\left([0, T] ; W^{\beta / 3,3}\right)$, then $X$ satisfies the energy equality on $[0, T]$.

Proof. Let $t \in[0, T]$ and $n \geq 1$. By equation (2),

$$
0 \leq E_{n}(t)+\int_{0}^{t} \sum_{i \leq n} \frac{2}{g_{i}} k_{i} X_{i}^{2}(u) d u=E_{n}(0)-\int_{0}^{t} 2 \phi_{n} k_{n} X_{n}^{2}(u) X_{n+1}(u) d u .
$$

To prove the energy equality, it is sufficient to take the limit for $n \rightarrow \infty$ and show that the last term of $\star$ converges to zero. By Young's inequality,

$$
\int_{0}^{t} k_{n} X_{n}^{2}(u)\left|X_{n+1}(u)\right| d u \leq \frac{2}{3} \int_{0}^{t} k_{n}\left|X_{n}(u)\right|^{3} d u+\frac{1}{3} \int_{0}^{t} k_{n+1}\left|X_{n+1}(u)\right|^{3} d u,
$$


and the terms on the right-hand side converge to zero, since $X \in L^{3}\left([0, T] ; W^{\beta / 3,3}\right)$.

\subsection{Local existence and uniqueness.}

Proposition 4. Let $s>0$ and suppose $x \in H^{s}, g \in H^{-s}$. Then there exist $\eta>$ 0 , depending only on $\|x\|_{H^{s}}$, and a unique solution in the class $\mathcal{H}:=L^{\infty}\left([0, \eta] ; H^{s}\right)$.

Proof. In view of applying Banach's fixed point theorem, we introduce the operator $\mathcal{F}$ on $\mathcal{H}$ defined as follows. For all $n \geq 1$ and $t \in[0, \eta]$, let

$$
(\mathcal{F} V)_{n}(t):=x_{n} e^{-\frac{k_{n}}{g_{n}} t}+\int_{0}^{t} e^{-\frac{k_{n}}{g_{n}}(t-u)}\left[\phi_{n-1} k_{n-1} V_{n-1}^{2}(u)-\phi_{n} k_{n} V_{n}(u) V_{n+1}(u)\right] d u,
$$

so that $X$ is a solution if and only if it is a fixed point of $\mathcal{F}$. To apply Banach's fixed point theorem we must show that $\mathcal{F}$ maps some ball $B_{\mathcal{H}}(M):=\{v \in \mathcal{H}$ : $\left.\|v\|_{\mathcal{H}} \leq M\right\}$ into itself and that $\mathcal{F}$ is a contraction on the ball. To this end, we will often use that if $v \in \mathcal{H}$, then $\left|v_{n}(t)\right| \leq k_{n}^{-s}\|v\|_{\mathcal{H}}$ for all $t \geq 0, n \geq 1$.

We deal with the first requirement, so suppose $V \in B_{\mathcal{H}}(M)$. For all $n \geq 1$ and $t \in[0, \eta]$,

$$
\begin{aligned}
\left|(\mathcal{F} V)_{n}(t)\right| & \leq\left|x_{n}\right| e^{-\frac{k_{n}}{g_{n}} t}+\|\phi\|_{\ell^{\infty}} \int_{0}^{t} e^{-\frac{k_{n}}{g_{n}}(t-u)}\left[k_{n-1} V_{n-1}^{2}+k_{n}\left|V_{n} V_{n+1}\right|\right] d u \\
& \leq\left|x_{n}\right| e^{-\frac{k_{n}}{g_{n}} t}+\|\phi\|_{\ell^{\infty}}\left(k_{n-1}^{1-2 s}+k_{n}^{1-s} k_{n+1}^{-s}\right)\|V\|^{2} \int_{0}^{t} e^{-\frac{k_{n}}{g_{n}}(t-u)} d u \\
& \leq\left|x_{n}\right|+2\|\phi\|_{\ell \infty} k_{n-1}^{-2 s} g_{n}\left(1-e^{-\frac{k_{n}}{g_{n}} t}\right)\|V\|^{2},
\end{aligned}
$$

so $\|\mathcal{F} V\|_{\mathcal{H}} \leq\|x\|_{H^{s}}+2\|\phi\|_{\ell^{\infty}}\|V\|^{2} L(\eta)$, where we defined

$$
L(t):=\left[\sum_{n \geq 1} k_{n}^{2 s} k_{n-1}^{-4 s} g_{n}^{2}\left(1-e^{-\frac{k_{n}}{g_{n}} t}\right)^{2}\right]^{1 / 2},
$$

and $\sup _{0 \leq t \leq \eta} L(t)=L(\eta)$ by monotonicity. We claim that $\lim _{\eta \rightarrow 0} L(\eta)=0$. Consider

$L^{2}(\eta)=2^{4 \beta s} \sum_{n \geq 1} k_{n}^{-2 s} g_{n}^{2}\left(1-e^{-\frac{k_{n}}{g_{n}} \eta}\right)^{2} \leq 2^{4 \beta s} \sum_{n=1}^{N} k_{n}^{-2 s} g_{n}^{2}\left(\frac{k_{n}}{g_{n}} \eta\right)^{2}+2^{4 \beta s} \sum_{n>N} k_{n}^{-2 s} g_{n}^{2}$.

Since $g \in H^{-s}$, we can choose $N$ such that the second term is arbitrarily small, and then choose $\eta$ in such a way that the first term is small too, hence $L(\eta) \rightarrow 0$ as $\eta \rightarrow 0$.

Let $M:=2\|x\|_{H^{s}}$. If $\eta$ is small enough so that $L(\eta) \leq\left(4\|\phi\|_{\ell^{\infty}} M\right)^{-1}$, then $\|\mathcal{F} V\|_{\mathcal{H}} \leq M / 2+2\|\phi\|_{\ell^{\infty}} M^{2} L(\eta) \leq M$, so the first requirement is satisfied for all $\eta$ such that $L(\eta) \leq\left(8\|\phi\|_{\ell^{\infty}}\|x\|_{H^{s}}\right)^{-1}$.

To prove that $\mathcal{F}$ is a contraction, suppose $X, Y \in B_{\mathcal{H}}(M)$. For all $n \geq 1$ and $t \in[0, \eta]$

$\left|(\mathcal{F} X-\mathcal{F} Y)_{n}(t)\right| \leq\|\phi\|_{\ell^{\infty}} \int_{0}^{t} e^{-\frac{k_{n}}{g_{n}}(t-u)}\left[k_{n-1}\left|X_{n-1}^{2}-Y_{n-1}^{2}\right|+k_{n}\left|X_{n} X_{n+1}-Y_{n} Y_{n+1}\right|\right] d u$. 
With the obvious decomposition $a b-c d=\frac{1}{2}(a-c)(b+d)+\frac{1}{2}(b-d)(a+c)$ and recalling that for all $j,\left|v_{j}(t)\right| \leq k_{j}^{-s}\|v\|_{\mathcal{H}}$, we get

$$
\begin{aligned}
\left|(\mathcal{F} X-\mathcal{F} Y)_{n}(t)\right| & \leq 2 M\|\phi\|_{\ell^{\infty}}\|X-Y\|_{\mathcal{H}}\left[k_{n-1} k_{n-1}^{-2 s}+k_{n} k_{n}^{-s} k_{n+1}^{-s}\right] \int_{0}^{t} e^{-\frac{k_{n}}{g_{n}}(t-u)} d u \\
& \leq 4 M\|\phi\|_{\ell^{\infty}}\|X-Y\|_{\mathcal{H}} k_{n-1}^{-2 s} g_{n}\left(1-e^{-\frac{k_{n}}{g_{n}} t}\right),
\end{aligned}
$$

hence

$$
\|\mathcal{F} X-\mathcal{F} Y\|_{\mathcal{H}} \leq 4 M\|\phi\|_{\ell \infty}\|X-Y\|_{\mathcal{H}} L(\eta) .
$$

Let $\theta \in(0,1)$. Choose $\eta$ small enough that $L(\eta) \leq \theta\left(8\|\phi\|_{\ell^{\infty}}\|x\|_{H^{s}}\right)^{-1}$. Then the first requirement is satisfied and $\|\mathcal{F} X-\mathcal{F} Y\|_{\mathcal{H}} \leq \theta\|X-Y\|_{\mathcal{H}}$, and we conclude by Banach's fixed point theorem.

\section{The main result}

In this section we prove our main result. The theorem follows immediately from our Theorem 13 , which works in a slightly more general setting.

THEOREM 5. Suppose that $g_{n}$ is non-decreasing, $\frac{k_{n}}{g_{n}}$ is eventually non-decreasing and that $\sum_{n \geq 1} g_{n}^{-1}=\infty$. If $x \in H^{s}$ for all $s>0$, then there exists a solution $X$ with initial condition $x$ such that $X \in L^{\infty}\left([0, \infty) ; H^{s}\right)$ for all $s>0$. This solution is unique in the class $L_{\mathrm{loc}}^{3}\left([0, \infty) ; W^{\beta / 3,3}\right)$.

3.1. The bounding sequence. For all initial condition in $H$, we introduce a sequence of positive numbers which will be fundamental to bound all weak solutions.

Definition 6. A sequence $y=\left(y_{n}\right)_{n \geq 1}$ is the bounding sequence for $x \in H$ if it is defined by

$$
\begin{aligned}
y_{1} & :=y_{2}:=2\|x\|_{H}^{2}, \\
y_{n+2} & :=C_{n+2}\left(y_{n+1}^{1 / 2}\right) y_{n}+\sum_{i \geq n+2} x_{i}^{2}, \quad n \geq 1,
\end{aligned}
$$

where for $n \geq 3, C_{n}: \mathbb{R}_{+} \mapsto(0,1)$ is the following increasing function,

$$
C_{n}(v):=\left(1+\frac{1}{\frac{1}{2} g_{n}\|\phi\|_{\ell \infty} v}\right)^{-1}, \quad v>0 .
$$

Lemma 7. Suppose $g$ is non-decreasing. Let $T>0, x \in H$ and $y$ be the bounding sequence for $x$. Suppose $X$ is a weak solution with initial condition $x$ that satisfies the energy inequality on $[0, t]$ for all $t<T$. Then $X_{n}^{2}(t) \leq y_{n}$ for all $t \in[0, T)$ and all $n \geq 1$.

Proof. Define

$$
d_{n}^{2}(t)=F_{n}(t)+\sum_{i \geq n+1} \int_{0}^{t} \frac{2}{g_{i}} k_{i} X_{i}^{2}(s) d s .
$$

Roughly speaking, $d_{n}^{2}$ represents the combination of the amount of energy stored in all modes larger than $n$ at time $t$ and the energy dissipated by these modes up to time $t$. 
Notice that $X_{n}^{2}(t) \leq F_{n}(t) \leq d_{n}^{2}(t) \leq\|x\|_{H}^{2}$ by the definition of $d_{n}$ and (3). By (4) we deduce that

$$
d_{n}^{2}(t) \leq \int_{0}^{t} 2 \phi_{n-1} k_{n-1} X_{n-1}^{2}(s) X_{n}(s) d s-\int_{0}^{t} \frac{2}{g_{n}} k_{n} X_{n}^{2}(s) d s+F_{n}(0) .
$$

Define

$$
\bar{d}_{n}:=\sup _{0 \leq t<T} d_{n}(t)<\infty .
$$

We claim that for all $n \geq 1$

$$
\bar{d}_{n+2}^{2} \leq C_{n+2}\left(\bar{d}_{n+1}\right) \bar{d}_{n}^{2}+F_{n+2}(0) .
$$

Then, since $y_{1}:=y_{2}:=2\|x\|_{H}^{2}$ and since $C_{n}$ is monotone increasing, an easy induction argument yields $\bar{d}_{n}^{2} \leq y_{n}$ for all $n \geq 1$ and hence that

$$
X_{n}^{2}(t) \leq d_{n}^{2}(t) \leq \bar{d}_{n}^{2} \leq y_{n},
$$

for all $n$ and all $t$.

We turn to the proof of the claim (9). By the Cauchy-Schwarz inequality applied to (8)

$$
\begin{gathered}
d_{n}^{2}(t) \leq \int_{0}^{t}\|\phi\|_{\ell \infty} k_{n-1}\left|X_{n-1}(s)\right|\left(X_{n-1}^{2}(s)+X_{n}^{2}(s)\right) d s+F_{n}(0) \\
\leq\|\phi\|_{\ell^{\infty}} \bar{d}_{n-1} \int_{0}^{t} k_{n-1}\left(X_{n-1}^{2}(s)+X_{n}^{2}(s)\right) d s+F_{n}(0) \\
\leq g_{n}\|\phi\|_{\ell^{\infty}} \bar{d}_{n-1} \int_{0}^{t}\left(\frac{k_{n-1}}{g_{n-1}} X_{n-1}^{2}(s)+\frac{k_{n}}{g_{n}} X_{n}^{2}(s)\right) d s+F_{n}(0),
\end{gathered}
$$

where we used the fact that $g_{n}$ and $k_{n}$ are non-decreasing with $n$. We get another bound from (7),

$$
\begin{aligned}
d_{n}^{2}(t)-d_{n-2}^{2}(t)=F_{n}(t)-F_{n-2}(t) & -\int_{0}^{t} \frac{2 k_{n-1}}{g_{n-1}} X_{n-1}^{2}(s) d s-\int_{0}^{t} \frac{2 k_{n}}{g_{n}} X_{n}^{2}(s) d s \\
& \leq-2 \int_{0}^{t}\left(\frac{k_{n-1}}{g_{n-1}} X_{n-1}^{2}(s)+\frac{k_{n}}{g_{n}} X_{n}^{2}(s)\right) d s
\end{aligned}
$$

hence putting the former into the latter,

$d_{n}^{2}(t) \leq d_{n-2}^{2}(t)-2 \int_{0}^{t}\left(\frac{k_{n-1}}{g_{n-1}} X_{n-1}^{2}(s)+\frac{k_{n}}{g_{n}} X_{n}^{2}(s)\right) d s \leq d_{n-2}^{2}(t)-\frac{d_{n}^{2}(t)-F_{n}(0)}{\frac{1}{2} g_{n}\|\phi\|_{\ell \infty} \bar{d}_{n-1}}$,

yielding

$$
d_{n}^{2}(t) \leq\left(1+\frac{1}{\frac{1}{2} g_{n}\|\phi\|_{\ell^{\infty}} \bar{d}_{n-1}}\right)^{-1} d_{n-2}^{2}(t)+F_{n}(0)=C_{n}\left(\bar{d}_{n-1}\right) d_{n-2}^{2}(t)+F_{n}(0) .
$$

Taking the sup for $s \in[0, T)$ yields the claimed inequality (9).

Lemma 7 states that the variables $X_{n}(t)$ can be bounded by the the bounding sequence $y$, so we will spend the rest of the section to show exponential decay for the bounding sequence $y_{n}$. As a first step we see that bounding sequences converge to 0 . 
LEMmA 8. Suppose $g$ is non-decreasing and $\sum_{n \geq 1} g_{n}^{-1}=\infty$. Let $x \in H^{s}$ for some $s>0$ and let $y$ be the bounding sequence for $x$. For all $n \geq 1$, let $h_{n}:=\sum_{j \geq n} \sum_{i \geq j} x_{i}^{2}$. Then

$$
y_{n+2 m} \leq y_{n} \prod_{i=1}^{m} C_{n+2 i}\left(y_{n+2 i-1}^{1 / 2}\right)+h_{n}, \quad \text { for all } n \geq 1, m \geq 0 .
$$

Moreover $y_{n} \rightarrow 0$ as $n \rightarrow \infty$.

Proof. Since $C_{j} \leq 1$ for all $j$, inequality 10 is easily proved by induction on $m$ using (6).

From this we deduce that $y$ is bounded. Since $v \mapsto C_{j}(v)$ is monotone increasing, we may replace the bound for $y$ inside $C_{j}$ yielding that

$$
C_{j}\left(y_{j-1}^{1 / 2}\right) \leq\left(1+c g_{j}^{-1}\right)^{-1}, \quad j \geq 1
$$

for some constant $c>0$. Since $\sum_{j \geq 1} g_{j}^{-1}=\infty$, then $\prod_{j \geq 1}\left(1+c g_{j}^{-1}\right)^{-1}=0$. Since $g$ is monotone, then $\prod_{i \geq 1}\left(1+c g_{n+2 i}^{-1}\right)^{-1}=0$ too, hence by considering 10 for $n$ and $n+1$, we get,

$$
\limsup _{j \geq n} y_{j} \leq h_{n}+h_{n+1} .
$$

Since $x \in H^{s}, \lim _{n \rightarrow \infty} h_{n}=0$, therefore $y_{n} \rightarrow 0$.

The next step is to introduce in Definition 9 below a special sub-sequence of the indices of $g_{n}$, this step is necessary because the hypothesis $\sum_{n} g_{n}=\infty$ does not provide enough information on the rate of divergence of the series.

DEFINITION 9. Given a sequence $g$ with $\sum_{n \geq 1} g_{n}^{-1}=\infty$, a positive integer $n_{0}$ and real numbers $\theta>0, s>0$, define by induction on $k \geq 0$,

$$
n_{k+1}:=\inf \left\{n \geq n_{k}+2: \sum_{j=n_{k}+2}^{n} g_{j}^{-1} \geq 2^{-s k} \theta\right\}<\infty .
$$

Notice that the definition above gives a finite number, because $\sum_{j \geq 1} g_{j}^{-1}=\infty$. The importance of this definition will be clear with the next two lemmas.

LEMmA 10. Suppose $g$ is non-decreasing and $\sum_{n>1} g_{n}^{-1}=\infty$. Let $x \in H^{s}$ for some $s>0$ and let $y$ be the bounding sequence for $x$. Then there exist $n_{0} \geq 1$ and $\theta>0$ such that the sequence $\left(n_{k}\right)_{k \geq 0}$ given in Definition 9 satisfies the following inequality:

$$
\sup _{j \geq n_{k}} y_{j} \leq 2^{-2 s k}, \quad k \geq 0 .
$$

PRoOF. In view of applying Lemma 8, we need to bound $y_{n}$ and $h_{n}$ for $n$ large. Since $x \in H^{s}$, then for any $\epsilon>0, x_{n} \leq \epsilon 2^{-s n}$ eventually and in particular for $n$ large,

$$
h_{n}=\sum_{j \geq n} \sum_{i \geq j} x_{i}^{2}=\sum_{j \geq 1} j x_{j+n-1}^{2} \leq 2^{-2 s n} \epsilon \sum_{j \geq 1} j 2^{-2 s(j-1)} .
$$

so for any $\eta>0, h_{n} \leq \eta 2^{-2 s n}$ eventually. We also know from Lemma 8 that $y_{n} \rightarrow 0$ as $n \rightarrow \infty$. Thus fix some $\eta>0$ and let $n_{0}$ be large enough that for all $n \geq n_{0}$,

$$
y_{n} \leq 1 \quad \text { and } \quad h_{n} \leq \eta 2^{-2 s n} .
$$

We now proceed to prove 12 by induction on $k \geq 0$. The initial step is simply given by the definition of $n_{0}$. 

$n_{k}+1$

We turn to the induction step. Suppose $\sup _{j \geq n_{k}} y_{j} \leq 2^{-2 s k}$. Then for $j \geq$

$$
C_{j}\left(y_{j-1}^{1 / 2}\right)^{-1}:=1+\frac{1}{\frac{1}{2} g_{j}\|\phi\|_{\ell^{\infty}} y_{j-1}^{1 / 2}} \geq 1+\frac{1}{\frac{1}{2} g_{j}\|\phi\|_{\ell^{\infty} 2^{-s k}}}=1+c 2^{s k} g_{j}^{-1},
$$

where $c=2 /\|\phi\|_{\ell \infty}$. By 10 we have then, for $n \geq n_{k}-1$,

$$
y_{n+2 m} \leq y_{n} \prod_{i=1}^{m}\left(1+c 2^{s k} g_{n+2 i}^{-1}\right)^{-1}+h_{n}
$$

By the monotonicity of $g$,

$$
\prod_{i=1}^{m}\left(1+c 2^{s k} g_{n+2 i}^{-1}\right) \geq 1+c 2^{s k} \sum_{i=1}^{m} g_{n+2 i}^{-1} \geq 1+c 2^{s k} \frac{1}{2} \sum_{j=n+2}^{n+2 m} g_{j}^{-1} .
$$

By the definition of $n_{k+1}$ in (11), if $n \leq n_{k}$ and $n+2 m \geq n_{k+1}$ we have

$$
\sum_{j=n+2}^{n+2 m} g_{j}^{-1} \geq 2^{-s k} \theta
$$

Collecting all conditions, we have proved that if $n \in\left\{n_{k}-1, n_{k}\right\}$ and $n+2 m \geq n_{k+1}$, then

$$
y_{n+2 m} \leq y_{n}\left(1+\frac{1}{2} c \theta\right)^{-1}+h_{n} .
$$

Since $n \geq n_{k}-1 \geq n_{k-1}$, then by inductive hypothesis $y_{n} \leq 2^{-2 s(k-1)}$; moreover since $n \geq n_{k}-1 \geq k$, then by the second one of $(13), h_{n} \leq \eta 2^{-2 s k}$, so the bound above becomes

$$
y_{n+2 m} \leq 2^{-2 s(k-1)}\left(1+\frac{1}{2} c \theta\right)^{-1}+\eta 2^{-2 s k} .
$$

Now we choose $\theta$ large enough and $\eta$ small enough that

$$
2^{2 s}\left(1+\frac{1}{2} c \theta\right)^{-1}+\eta \leq 2^{-2 s}
$$

to get

$$
y_{n+2 m} \leq 2^{-2 s(k+1)}, \quad n \in\left\{n_{k}-1, n_{k}\right\}, \quad n+2 m \geq n_{k+1} .
$$

Since for all $j \geq n_{k+1}$ there exist $n$ and $m$ such that $n_{k}-1 \leq n \leq n_{k}$ and $j=n+2 m \geq n_{k+1}$, we have proved

$$
\sup _{j \geq n_{k+1}} y_{j} \leq 2^{-2 s(k+1)},
$$

closing the induction.

Lemma 11. Suppose $g$ is non-decreasing and $\sum_{n \geq 1} g_{n}^{-1}=\infty$. Let $n_{0} \geq 1$ and $\theta>0$ be constant. If $\left(n_{k}\right)_{k \geq 0}$ is as in Definition 9 then there exist infinitely many $k \geq 1$ such that $n_{k+1}=n_{k}+2$.

Proof. Suppose that there exists a non-negative integer $r$ such that $n_{k+1} \geq$ $n_{k}+3$ for all $k \geq r$. By the definition of the sequence $\left(n_{k}\right)_{k \geq 0}$, we know that for $k \geq r$

$$
\sum_{j=n_{k}+2}^{n_{k+1}-1} g_{j}^{-1}<2^{-s k} \theta
$$


Summing on $k$ we obtain

$$
\sum_{k \geq r} \sum_{j=n_{k}+2}^{n_{k+1}-1} g_{j}^{-1}<\infty
$$

hence since $\sum_{j \geq n_{r}} g_{j}^{-1}=\infty$,

$$
\sum_{k \geq r}\left(g_{n_{k}}^{-1}+g_{n_{k}+1}^{-1}\right)=\infty .
$$

But $g_{n_{k}+1}^{-1} \leq g_{n_{k}}^{-1} \leq g_{n_{k-1}+2}^{-1} \leq 2^{-s(k-1)} \theta$, which is in contradiction with 14. Hence there exist infinitely many $k$ such that $n_{k+1}=n_{k}+2$.

Lemma 12. Let $x \in H^{s}$ for some $s>0$ and let $y$ be the bounding sequence for $x$. Suppose that $g_{n}$ is non-decreasing, $g_{n} 2^{-s n}$ is eventually non-increasing and that $\sum_{n \geq 1} g_{n}^{-1}=\infty$. Then

$$
\sum_{n \geq 1} 2^{2 s n} y_{n}<\infty
$$

Proof. Let us recall the recursion (6) that defines the bounding sequence $y$,

$$
y_{n+2}:=c_{n+2} y_{n}+f_{n+2}, \quad n \geq 1,
$$

where

$$
c_{n}:=C_{n}\left(y_{n-1}^{1 / 2}\right):=\left(1+\frac{1}{\frac{1}{2} g_{n}\|\phi\|_{\ell_{\infty}} y_{n-1}^{1 / 2}}\right)^{-1}, \quad n \geq 3,
$$

and where $f_{n}:=F_{n}(0), n \geq 3$. Since $x \in H^{s}$, it is immediate that

$$
\sum_{n \geq 1} 2^{2 s n} f_{n}<\infty
$$

so our strategy will be to show that $c_{n} \rightarrow 0$ as $n \rightarrow \infty$.

By Lemma 10 there exist $n_{0}$ and $\theta$ such that the sequence $\left(n_{i}\right)_{i \geq 1}$ of Definition 9 satisfies

$$
\sup _{j \geq n_{i}} y_{j} \leq 2^{-2 s i}, \quad i \geq 0 .
$$

A fortiori these inequalities hold also if we take larger values for $n_{0}$ and $\theta$, so let $\theta$ be large enough to verify inequality 20 below and let $n_{0}$ be large enough that:

(1) $f_{n} \leq \frac{1}{2} 2^{-2 s n}$ for $n \geq n_{0}$ (a consequence of 16 );

(2) $n \mapsto g_{n} 2^{-s n}$ is non-increasing for $n \geq n_{0}$. hence

By Lemma 11 there exists $k$ such that $n_{k+1}=2+n_{k}$, that is, $g_{n_{k}+2}^{-1} \geq 2^{-s k} \theta$

$$
g_{n_{k}+m} \leq \frac{1}{2^{2 s} \theta} 2^{s(k+m)}, \quad m \geq 2 .
$$

We have all the ingredients to prove the following inequality:

$$
y_{n_{k}+m} \leq 2^{2 s} 2^{-2 s(k+m)}, \quad m \geq 0 .
$$

Let us proceed by induction on $m$. The initial steps for $m=0$ and $m=1$ follow immediately from (17) with $i=k$. 
For the inductive step, suppose the inequality 19 is true up to $m+1$. By 18 and the inductive hypothesis,

$$
c_{n_{k}+m+2}=\left(1+\frac{1}{\frac{1}{2} g_{n_{k}+m+2}\|\phi\|_{\ell^{\infty}} y_{n+k+m+1}^{1 / 2}}\right)^{-1} \leq\left(1+\frac{2 \theta}{\|\phi\|_{\ell^{\infty}}}\right)^{-1} \leq \frac{1}{2} 2^{-4 s},
$$

if we choose $\theta$ large enough that

$$
\left(1+\frac{2 \theta}{\|\phi\|_{\ell \infty}}\right)^{-1} \leq \frac{1}{2} 2^{-4 s}
$$

Moreover, since $n_{k} \geq k-1$, we have

$$
f_{n_{k}+m+2} \leq \frac{1}{2} 2^{-2 s\left(n_{k}+m+2\right)} \leq \frac{1}{2} 2^{2 s} 2^{-2 s(k+m+2)},
$$

hence

$$
y_{n_{k}+m+2}=c_{n_{k}+m+2} y_{n_{k}+m}+f_{n_{k}+m+2} \leq 2^{2 s} 2^{-2 s(k+m+2)},
$$

thus closing the induction.

Inequality (19) says us that $y_{n} \rightarrow 0$ at least as fast as $2^{-2 s n}$. To get $\sum_{n} 2^{2 s n} y_{n}<$ $\infty$ we need a little bit more. We proved above that for any $\theta$ large enough there exists $n_{k}$ such that

$$
\sup _{j \geq n_{k}} c_{j} \leq\left(1+\frac{2 \theta}{\|\phi\|_{\ell \infty}}\right)^{-1}
$$

By the arbitrarity of $\theta, \lim _{n \rightarrow \infty} c_{n}=0$. This together with 15 and 16 proves that

$$
\sum_{n \geq 1} 2^{2 s n} y_{n}<\infty
$$

\subsection{Global existence, uniqueness and regularity.}

Theorem 13. Let $x \in H^{s}$ for some $s>\frac{\beta}{3}$. Suppose that $g_{n} \in H^{-s}$ is nondecreasing, $g_{n} 2^{-s n}$ is eventually non-increasing and that $\sum_{n \geq 1} g_{n}^{-1}=\infty$. Then there exists a solution in the class $L^{\infty}\left([0, \infty) ; H^{s}\right)$. This solution is unique in the class $L_{\mathrm{loc}}^{3}\left([0, \infty) ; W^{\beta / 3,3}\right)$.

Proof. Let $T>0$ be the maximal time of existence in $H^{s}$ of the solution provided by Proposition 4 . In particular, $X \in L^{\infty}\left([0, t] ; H^{s}\right)$ for all $t<T$ and, since $s>\beta / 3, X \in L^{3}\left([0, t] ; W^{\beta / 3,3}\right)$ for all $t<T$. Hence, by Proposition $3, X$ satisfies the energy equality on $[0, t]$. Lemma 7 applies, so if $y$ denotes the bounding sequence for $x$, we have

$$
X_{n}^{2}(t) \leq y_{n}, \quad n \geq 1, \quad t \in[0, T) .
$$

By Lemma 12

$$
\sup _{t \in[0, T)}\|X(t)\|_{H^{s}}^{2} \leq \sum_{n \geq 1} 2^{2 s n} y_{n}<\infty .
$$

If $T=\infty$ we just proved $X \in L^{\infty}\left([0, \infty) ; H^{s}\right)$. Suppose by contradiction that $T<\infty$. Then the bound in $(21)$ can be extended to $t \in[0, T]$ by the continuity of $X_{n}$ hence again by Lemma $12, X(T) \in H^{s}$ and it would be possible to apply Proposition 4 in contradiction with the maximality of $T$.

Finally we turn to uniqueness in $L_{\text {loc }}^{3}\left([0, \infty) ; W^{\beta / 3,3}\right)$. By Proposition 3 . Lemma 7 and Lemma 12 , if $X$ is a solution of class $L_{\text {loc }}^{3}\left([0, \infty) ; W^{\beta / 3,3}\right)$, then $X$ is also of class $L_{\mathrm{loc}}^{\infty}\left([0, \infty) ; H^{s}\right)$, hence by Proposition 4 it is unique. 
3.3. Additional remarks. The last part of the paper is devoted to some final remarks about our results. They have been collected here in order to give a more complete understanding of the problem, while focusing, in the main body of the paper, on the assumptions corresponding to those of [13].

3.3.1. A useful generalization. The results presented in the previous sections allow for more general coefficients $\phi$. Namely, assume that

$$
\phi_{n}=\phi_{n}\left(t, X_{n-m}, X_{n-m+1}, \ldots, X_{n+m}\right),
$$

for all $n \geq 1$, where $m \geq 1$ is a fixed integer. For convenience we set $X_{-m}=$ $X_{-m+1}=\cdots=X_{0}=0$. Assume moreover that the functions $\left(\phi_{n}\right)_{n \geq 1}$ are uniformly bounded and uniformly Lipschitz. This ensures that the local existence and uniqueness theorem (Proposition 4 still holds. In Proposition 3 and Lemma 7 we only use the uniform boundedness, while lemmas 8, 10,12 deal only with bounding sequences.

The above model has a nice application to the averaged Navier-Stokes system studied by Tao in 14. By making a special average on the transport of the NS equations, the author derives a vector-valued dyadic system, very similar to (1) but with four components for each $n$. A general version of this averaged system is

$$
\left\{\begin{array}{l}
X_{1, n}^{\prime}=-\frac{k_{n}^{\alpha}}{g_{n}} X_{1, n}+k_{n}^{\gamma}\left(-C_{1} X_{3, n} X_{4, n}-C_{2} X_{1, n} X_{2, n}-C_{3} X_{1, n} X_{3, n}+C_{4} X_{4, n-1}^{2}\right) \\
X_{2, n}^{\prime}=-\frac{k_{n}}{g_{n}} X_{2, n}+k_{n}^{\gamma}\left(C_{2} X_{1, n}^{2}-C_{5} X_{3, n}^{2}\right) \\
X_{3, n}^{\prime}=-\frac{k_{n}^{\alpha}}{g_{n}} X_{3, n}+k_{n}^{\gamma}\left(C_{3} X_{1, n}^{2}+C_{5} X_{2, n} X_{3, n}\right) \\
X_{4, n}^{\prime}=-\frac{k_{n}^{n}}{g_{n}} X_{4, n}+k_{n}^{\gamma} C_{1} X_{1, n} X_{3, n}-k_{n+1}^{\gamma} C_{4} X_{4, n} X_{1, n+1} \\
X_{\cdot, n}(0)=x \cdot, n
\end{array}\right.
$$

for all $t>0$ and $n \geq 1$.

Here $X=\left(X_{i, n}\right)_{i \in\{1,2,3,4\}, n \geq 1}$ is a family of real functions, $X_{i, n}:[0, \infty) \rightarrow \mathbb{R}$; $X_{\cdot, 0} \equiv 0 ; x=\left(x_{i, n}\right)_{i \in\{1,2,3,4\}, n \geq 1}$ is the given initial condition, $k_{n}=2^{\beta n}$ with $\beta>0$, and $C_{1}, \ldots, C_{5}$ are five real constants.

In the framework of Navier-Stokes equations the constants $\alpha=2$ and $\gamma=\frac{5}{2}$ give a strictly supercritical regime. In $\mathbf{1 4}$ the author shows that this system with a suitable initial condition develops a singularity. For the system $(23)$ the critical regime is for $\alpha=\gamma$ and $g \equiv 1$ (it is the regime in which the transport effects are of the same order of the dissipative effect) whereas the logarithmically supercritical regime conjectured in $\left[\mathbf{1 3}\right.$ is given by $\alpha=\gamma$ and $g$ such that $\sum_{n} g_{n}^{-1}=\infty$.

The latter case can be included in our model (1) with general coefficients (22). Indeed, by summing up the components $X_{n}^{2}:=\sum_{i=1}^{4} X_{i, n}^{2}$ one gets

$$
\begin{aligned}
\frac{1}{2} \frac{d}{d t} X_{n}^{2} & =-\frac{k_{n}^{\alpha}}{g_{n}} X_{n}^{2}+C_{4}\left(k_{n}^{\gamma} X_{4, n-1}^{2} X_{1, n}-k_{n+1}^{\gamma} X_{4, n}^{2} X_{1, n+1}\right) \\
& =-\frac{k_{n}^{\alpha}}{g_{n}} X_{n}^{2}+\phi_{n} k_{n}^{\gamma} X_{n-1}^{2} X_{n}-\phi_{n+1} k_{n+1}^{\gamma} X_{n}^{2} X_{n+1}
\end{aligned}
$$

and this can be reduced to the system (1), when $\alpha=\gamma$ by a suitable choice of $\beta$ and appropriate functions $\phi_{n}(t)$ depending on $n$ and $t$ and uniformly bounded.

3.3.2. Conditions for smoothing. Here we study the smoothing effect of the dissipative part. We work under the assumptions of Theorem 5 .

The linear operator. Consider the system $Z_{n}^{\prime}=-\frac{k_{n}}{g_{n}} Z_{n}, n \geq 1$, the linear part of (1). 
Lemma 14. Assume additionally that $\frac{n g_{n}}{k_{n}} \rightarrow 0$. If $x \in \ell^{2}$ and $Z$ is the solution starting at $x$, then $Z(t) \in L^{\infty}\left([\epsilon, \infty) ; H^{s}\right)$ for every $\epsilon>0$ and every $s>0$.

Proof. Clearly $Z_{n}(t)=x_{n} \exp \left(-\frac{k_{n}}{g_{n}} t\right)$ and $\sup _{n}\left(2^{s n} Z_{n}(t)\right)<\infty$ for all $s>0$, $t>0$ if and only if $\frac{n g_{n}}{k_{n}} \rightarrow 0$.

REMARK 15. If $\frac{n g_{n}}{k_{n}} \nrightarrow 0$, the linear dissipation may not have a smoothing effect. Indeed, it is easy to construct a counterexample. Choose $n_{1} \geq 1$ and set $n_{p+1}=n_{p} 2^{\beta k_{n_{p}} / n_{p}}, p \geq 1, g_{n_{p}}=k_{n_{p}} / n_{p}$, and define $g_{n}=g_{n_{p}}$ for $n_{p} \leq n \leq m_{p}$, and $g_{n}=g_{n_{p}} k_{n} / k_{m_{p}}$ for $m_{p}+1 \leq n<n_{p+1}$, where $m_{p}=n_{p}+k_{n_{p}} / n_{p}$ It is easy to verify that $\left(g_{n}\right)_{n \geq 1}$ satisfies our standing assumptions and that there are sequences $\left(x_{n}\right)_{n \geq 1} \in \ell^{2}$ such that the corresponding solution $Z$ is not smooth.

Smoothing by dissipation. We now analyse the smoothing effect for the nonlinear equation. Our final result is the following.

TheOrem 16. Assume additionally that $\frac{n g_{n}}{k_{n}} \rightarrow 0$. If $s>\beta$ and $X$ is a solution such that $X(0) \in H^{s}$ and $X \in L^{\infty}\left([0, T] ; H^{s}\right)$, then $X \in L_{\mathrm{loc}}^{\infty}\left((0, T] ; H^{s}\right)$ for every $s>0$.

The theorem follows immediately from the following lemma.

LEMMA 17. Under the same assumptions of the previous theorem, let $s_{1}>\beta$ and $s_{2} \in\left(s_{1}, 2 s_{1}-\beta\right)$. If $X$ is a solution such that $X(0) \in H^{s_{1}}$ and $X \in$ $L^{\infty}\left([0, T] ; H^{s_{1}}\right)$, then $X \in L_{\mathrm{loc}}^{\infty}\left((0, T] ; H^{s_{2}}\right)$. More precisely, there is a non-decreasing upper semi-continuous function $\varphi:(0, \infty) \rightarrow \mathbf{R}$ such that $\varphi$ is continuous in 0 with $\varphi(0)=0$, and

$$
\sup _{t \in[0, T]}\left(\varphi(t)\|X(t)\|_{H^{s_{2}}}\right)<\infty .
$$

Proof. We have that

$2^{s_{2} n} X_{n}(t)=2^{s_{2} n} \mathrm{e}^{-\frac{k_{n}}{g_{n}} t} X_{n}(0)+2^{s_{2} n} \int_{0}^{t} \mathrm{e}^{-\frac{k_{n}}{g_{n}}(t-s)}\left(\phi_{n-1} k_{n-1} X_{n-1}^{2}-\phi_{n} k_{n} X_{n} X_{n+1}\right) d s$,

and consider the two terms on the right hand side separately.

For the non-linear term, we use the inequality $\left|X_{n}(t)\right| \leq 2^{-s_{1} n}\|X\|_{L^{\infty}\left(H^{s_{1}}\right)}$ to get

$$
\begin{aligned}
& \left|2^{s_{2} n} \int_{0}^{t} \mathrm{e}^{-\frac{k_{n}}{g_{n}}(t-s)}\left(\phi_{n-1} k_{n-1} X_{n-1}^{2}-\phi_{n} k_{n} X_{n} X_{n+1}\right) d s\right| \leq \\
& \quad \leq c\|X\|_{L^{\infty}\left(H^{s_{1}}\right)}^{2}\|\phi\|_{\ell^{\infty} 2^{n\left(s_{2}-2 s_{1}\right)}} g_{n} \leq c\|X\|_{L^{\infty}\left(H^{s_{1}}\right)}^{2}\|\phi\|_{\ell^{\infty}} 2^{n\left(s_{2}-2 s_{1}+\beta\right)} \in \ell^{2},
\end{aligned}
$$

since $g_{n} \leq c k_{n}$ and, by the choice of $s_{2}, s_{2}-2 s_{1}+\beta<0$.

For the term with the initial condition we notice that

$$
2^{s_{2} n} \mathrm{e}^{-\frac{k_{n}}{g_{n}} t}\left|X_{n}(0)\right|=2^{\left(s_{2}-s_{1}\right) n} \mathrm{e}^{-\frac{k_{n}}{g_{n}} t}\left(2^{s_{1} n}\left|X_{n}(0)\right|\right) \leq \psi(t)\left(2^{s_{1} n}\left|X_{n}(0)\right|\right) \in \ell^{2},
$$

where $\psi(t)=\sup _{n}\left(2^{\left(s_{2}-s_{1}\right) n} \exp \left(-\frac{k_{n}}{g_{n}} t\right)\right)$. It is easy to check that $\psi$ is nonincreasing, lower semi-continuous and $\psi(t) \uparrow \infty$ as $t \downarrow 0$. Choose $\varphi=1 / \psi$ to conclude the proof. 


\section{References}

[1] David Barbato, Franco Flandoli, and Francesco Morandin. A theorem of uniqueness for an inviscid dyadic model. C. R. Math. Acad. Sci. Paris, 348(9-10):525-528, 2010.

[2] David Barbato, Franco Flandoli, and Francesco Morandin. Energy dissipation and self-similar solutions for an unforced inviscid dyadic model. Trans. Amer. Math. Soc., 363(4):1925-1946, 2011.

[3] David Barbato and Francesco Morandin. Positive and non-positive solutions for an inviscid dyadic model: well-posedness and regularity. NoDEA Nonlinear Differential Equations Appl., 20(3):1105-1123, 2013.

[4] David Barbato, Francesco Morandin, and Marco Romito. Smooth solutions for the dyadic model. Nonlinearity, 24(11):3083-3097, 2011.

[5] A. Cheskidov and S. Friedlander. The vanishing viscosity limit for a dyadic model. Physica D: Nonlinear Phenomena, 238(8):783-787, 2009.

[6] Alexey Cheskidov. Blow-up in finite time for the dyadic model of the Navier-Stokes equations. Trans. Amer. Math. Soc., 360(10):5101-5120, 2008.

[7] Alexey Cheskidov, Susan Friedlander, and Nataša Pavlović. Inviscid dyadic model of turbulence: the fixed point and Onsager's conjecture. J. Math. Phys., 48(6):065503, 16, 2007.

[8] Alexey Cheskidov, Susan Friedlander, and Nataša Pavlović. An inviscid dyadic model of turbulence: the global attractor. Discrete Contin. Dyn. Syst., 26(3):781-794, 2010.

[9] N. H. Katz and N. Pavlović. A cheap Caffarelli-Kohn-Nirenberg inequality for the NavierStokes equation with hyper-dissipation. Geom. Funct. Anal., 12(2):355-379, 2002.

[10] Nets Hawk Katz and Nataša Pavlović. Finite time blow-up for a dyadic model of the Euler equations. Trans. Amer. Math. Soc., 357(2):695-708 (electronic), 2005.

[11] J. C. Mattingly and Ya. G. Sinai. An elementary proof of the existence and uniqueness theorem for the Navier-Stokes equations. Commun. Contemp. Math., 1(4):497-516, 1999.

[12] Terence Tao. Global regularity for a logarithmically supercritical defocusing nonlinear wave equation for spherically symmetric data. J. Hyperbolic Differ. Equ., 4(2):259-265, 2007.

[13] Terence Tao. Global regularity for a logarithmically supercritical hyperdissipative NavierStokes equation. Anal. PDE, 2(3):361-366, 2009.

[14] Terence Tao. Finite time blowup for an averaged three-dimensional Navier-Stokes equation, 2014. arXiv:1402.0290.

UniVersità Di PADOVA

E-mail address: barbato@math.unipd.it

Università di PARMA

E-mail address: francesco.morandin@unipr.it

UNIVERSITÀ DI PISA

E-mail address: romito@dm.unipi.it 